\title{
REVISIÓN SISTEMÁTICA ACERCA DE LAS TENDENCIAS INVESTIGATIVAS DEL SEXTING CONSENSUADO EN EL MARCO DE LA CIBERSEXUALIDAD ADOLESCENTE
}

\author{
Cynthia Sanhueza Cornejos \\ Universidad LiberQuaré \\ cynthiasanhueza@hotmail.com
}

Recepción Artículo: 10 junio 2021

Admisión Evaluación: 10 junio 2021

Informe Evaluador 1: 12 junio 2021

Informe Evaluador 2: 13 junio 2021

Aprobación Publicación: 14 junio 2021

\section{RESUMEN}

El presente trabajo tiene por objetivo revisar la literatura disponible sobre sexting adolescente consensuado. Se realizó mediante la revisión sistemática evidenciada en la literatura científica y contenida en la base de datos Pubmed, durante el período 2019 y 2020. Se encontraron 4 artículos, la mayoría de ellos desarrollados en Estados Unidos. El nivel de evidencia científica oscila entre buena y moderada (grados I.a y II.b), procedentes de estudios controlados y cuasiexperimentales y el grado de recomendación fue B. Las categorías encontradas corresponden a la posible relación entre la sexualidad virtual normativa y dual, los impactos positivos del sexting consensuado y los grados de satisfación sexual, afectividad y aceptación que presentan los/as adolescentes que lo practican.

Palabras clave: sexting; adolescentes; sexting concensuado

\section{ABSTRACT}

Systematic review of research trends on consensual sexting in the context of adolescent cybersexuality. This article offers a review of the available literature on consensual adolescent sexting. It was carried out by a systematic analysis of the scientific literature contained in the Pubmed database, between the years 2019 to 2020. Four articles were found, most of them written in the United States. The level of scientific evidence ranged from good to moderate (grades I.a and II.b), from controlled and quasi-experimental studies and the grade of recommendation was $B$. The categories found correspond to the possible relationship between normative and dual virtual sexuality, the positive impacts of consensual sexting and the degrees of sexual satisfaction, affection and acceptance shown by adolescents who practice it.

Keywords: sexting; adolescents; consensual sexting 


\section{REVISIÓN SISTEMÁTICA ACERCA DE LAS TENDENCIAS INVESTIGATIVAS DEL SEXTING CONSENSUADO EN EL MARCO DE LA CIBERSEXUALIDAD ADOLESCENTE}

\section{INTRODUCCIÓN}

El sexting, generalmente es definido como el envío, recepción o reenvío de contenidos eróticos o sexuales (mensajes, fotos o vídeos), a través de dispositivos tecnológicos interactivos, principalmente móviles. [1,2]

La velocidad con la cual aumentan y se diversifican los recursos de transmisión y comunicación virtual, empujan a los sujetos hacia la adquisición de nuevos modos de relacionarse y de establecer puntos de coincidencias en la relaciones intersujetivas. El sexting es, precisamente, un intercambio que se da en la esfera de la intimidad. Y la intimidad es un valor que forma parte de nuestros derechos y libertades. Sin embargo, cuando esa intimidad se vulnera como consecuencia de la divulgación sin consentimiento de los contenidos visuales, la culpabilidad recae generalmente hacia quien genera la imagen y no necesariamente hacia quien la distribuye de forma indebida. [3].

Por esta razón es que comúnmente el sexting se asocia a un "problema" que podría perjudicar a quienes lo practican. De hecho las investigaciones tienden a relacionarlo con otras conductas de riesgo como por ejemplo; a consumo de drogas 0 alcohol, situaciones de violencia en las relaciones de pareja, infidelidad, agresión sexual o victimización por acoso cibernético [4,5] así como asociaciones con problemas de salud mental $[6,7,8]$

Debido a lo anterior la estigmatización del sexting es una respuesta esperada y "natural" ante un fenómeno que desborda el ámbito sociocultural y científico actual. Como relato en el imaginario moderno, el sexting puede producir curiosidad, rechazo, repulsión y/o sospecha.

Frente este tipo de "amenaza" lo que se estila es una especie de "prohibicionismo" que en vez de neutralizar esta práctica, lo que hace directamente es reproducir patrones y modelos de relaciones de violencia y acoso patriarcal, machista y de género.

Es por ello que se hace necesario fortalecer la comprensión del fenómeno con el fin de diseñar estrategias de actuación ante estas nuevas formas de sexualidad que irrumpen culturalmente y que adquieren mayor arriago en las nuevas generaciones.

De manera con esta revisión sistemática se discute el significado del sexting consensuado en adolescentes a la luz de las investigaciones más recientes.

El sexting consensuado se puede entender genéricamente como aquella relación de reciprocidad en el intercambio (envío, recepción y respuesta) [9] entre parejas de imágenes, mensajes, fotos o videos con contenido erótico y sexual, que podría no generar ningún tipo de riesgo puesto que la decisión de llevarla adelante no depende de alguna acción coercitiva

Existen estudios que señalan que el sexteo consensuado en general, se asocia con una mayor satisfacción sexual y satisfacción en la relación. [10]. Particularmente y tal como lo señala Cornelius, et.al [11] para los adolescentes tardíos, el sexting consensuado sería beneficioso en la consolidación de su sexualidad puesto que las expectativas asocicadas a este son positivas con más frecuencia que las expectativas negativas y a menudo citaron motivaciones como el coqueteo, la diversión, la exploración sexual y la iniciación sexual.

Por otra parte, se puede entender la sexualidad virtual normativa como la construcción de una nueva identidad [12] sexual en el /la joven, en este caso basada en el manejo expandido de las nuevas tecnologías de comunicación. La que se encuentra modelada en torno a ideales externos de perfección, éxito y estándares de belleza que están separadas o disociadas entre las realidades sociales y psicológicas concretas y las virtuales del sujeto , constituyendose en un discurso mayoritario y aceptado. [13].Y entre este discurso dominante y virtual, este tipo de sexualidad se acepta como una extensión normal del desarrollo sexual.

Se considera que esta sexualidad virtual normalizada es dual puesto que: La mayoría de los estudios cualitativos sobre discursos adolescentes sobre sexting revelan dos posiciones al respecto; por un lado quienes asumen el discurso dominante, los que aceptan los requerimientos de la sexualidad virtual, y por otro quienes objetan y definen sus posiciones como un rechazo a la sexualidad virtual 0 en línea con la cultura sexual tradicional fuera de línea. [14 p. 4.]

En este sentido se destacan los últimos esfuerzos desarrollados por los/as investigadores/as en relación a poder distinguir los efectos de estas prácticas consensuadas y no consensuadas [15]. 
Es por ello que mediante esta revisión sistemática e identificación de las tendencias de investigación se pretende indagar en los elementos conceptuales y operativos del sexting consensuado en la cibersexualidad adolescente.

Además, se reconcerá el nivel de evidencia y recomendación de los artículos seleccionados. De esta forma se espera contribuir al conocimiento científico, mediante la revisión de literatura actualizada y generar futuras investigaciones con una perspectiva crítica.

El análisis de la investigación arrojó resultados que propiciaron la estructuración de 3 categorías que buscaron enmarcar las temáticas más representativas para los investigadores asociados a sexting adolescente, y la evidencia de sus correspondientes tendencias.

Para esta revisión sistemática se contempló el período comprendido entre el año 2019-2020 en la literatura científica contenida en la base de datos Pubmed Finalmente, se seleccionaron 4 artículos, desarrollados en su mayoría en Estados Unidos.

El grado de recomendación fue B y el nivel de evidencia fue moderado entre la y III.

El tipo de estudio predominante fue el empírico, además uno corresponde a revisión documental (metanálisis) y otro de tipo mixta y el muestreo de tipo intencional aleatorio.

\section{MÉTODO}

Tal como lo señala Villada [16] la revisión sistemática, es un trabajo de síntesis que pretende reportar el nivel de evidencia que presentan las investigaciones sobre un tema particular en un período de tiempo limitado, permitiendo descubrir tendencias investigativas.

La revisión que aquí se presenta se realizó siguiendo los siete pasos del modelo de Cooper [17] con el objetivo de asegurar la calidad de los estudios 1). La formulación del problema, 2). Búsqueda de literatura, 3). Reunión de información de los estudios, 4). Evaluación de la calidad de los estudios, 5). Análisis e integración de los resultados, 6). Interpretación de la evidencia y 7). Presentación de los resultados.

Para evaluar la calidad de los estudios se elaboró una tabla donde se incluyó: autor y año, nombre de la revista, rango de edad, el país donde se llevó a cabo, muestreo, tipo de instrumento, resultados, conclusiones, y la fiabilidad de los datos, es decir, el nivel de evidencia según el alcance del tipo de estudio y grado de recomendación.

Se utilizó para la selección de los artículos el Diagrama de flujo de QUORUM (Quality of Reporting of Metaanalyses), descrito en Moraga et.al [18]

La búsqueda inicial se realizó en la base de datos PubMed, para ello se filtraron aquellos artículos según las palabras clave: "sexting, sexting consensuado y adolescentes" que se usaron como los indicadores principales para la indagación de la información. Además, se examinaron las listas de referencias de los artículos revisados en relación con el tema de búsqueda. Por otra parte, la revisión se focalizó en la literatura publicada entre el año 2019- 2020, escrita en inglés o español, y publicada en revistas indexadas.

Como criterio de exclusión, no se consideraron aquellas publicaciones con población objeto mayor de 18 años, publicaciones anteriores al 2019 y artículos sin indexación. . Asimismo, boletines, reseñas de libros, comentarios o perspectivas que no se deriven de investigaciones.

La búsqueda en la base de datos produjo un total de 43 documentos. Se excluyeron 10 artículos que no cumplían con los criterios de selección como edad de la población objeto de estudio, relación con la temática a investigar, intervalo de tiempo considerado. Como resultado se obtuvieron 33 estudios para su revisión.

De estos 33 artículos, sólo 4 cumplieron los requisitos de tema de estudio, población objeto de estudio, período seleccionado (2019-2020) y temática enfocada en el tema de sexting consensuado y adolescencia, además de la indexación. 


\section{REVISIÓN SISTEMÁTICA ACERCA DE LAS TENDENCIAS INVESTIGATIVAS DEL SEXTING CONSENSUADO EN EL MARCO DE LA CIBERSEXUALIDAD ADOLESCENTE}

\section{RESULTADOS}

Para el presente trabajo se puede apreciar que de los 4 estudios todos fueron en idioma inglés, con muestras de adolescentes entre los 13 y 18 años. Las revistas de los estudios seleccionados tienen un factor de impacto entre 3.131 y 2.468, con una media de 2.79 .

\section{Tipos de estudio}

Se detectó en este grupo de documentos el predominio de estudios de tipo empírico, lo que representa el $50 \%$ del total. Esta cifra obtiene una alta ponderación comparando los resultados de los estudios de tipo revisión documental (25\%) y mixta (25\%). En este punto es necesario enfatizar que dentro de la totalidad de los artículos revisados uno se comprometió en analizar el sexting consensuado y no consensuado, utilizando la revisión sistemática. [2]. Este hecho destaca por cuanto presenta una perspectiva en la cual, si bien predominan los estudios de tipo empíricos, hay un avance hacia la discusión teórica entendiendo la complejidad de las distintas esferas que están presentes en el fenómeno del cybersexualismo y adolescencia.

Dentro de los estudios empíricos destacan aquellos vinculados a la categoría de "Sexting consensuado y reciproco relacionado con la satisfación sexual, afectividad y aceptación", con una frecuencia de 1 publicación. [2]. Así mismo, del tipo documental la encontramos en la categoría "Integración en la sexualidad adolescente de los impactos positivos del sexting consensuado" que está representada en dos publicaciones [14,19]. Y finalmente, la categoría de "Sexualidad virtual normativa y dual" se encuentra vinculada al tipo mixto, con una publicación indexada. [11]

Tabla II Tipos de Estudio

\begin{tabular}{lcc} 
Tipo & Frecuencia & \% \\
\hline Empirico & 2 & 50 \\
\hline Documental & 1 & 25 \\
\hline Mixto & 1 & 25 \\
\hline Total & 4 & $\mathbf{1 0 0} \%$
\end{tabular}

\section{Diseño de estudio}

En cuanto al diseño de estudio, los datos indican que prevalecen los estudios que en su diseño conllevan la descripción y análisis de datos obtenidos. Utilizado para ello recursos de procesamiento estadístico y matemático, toda vez que la obtención de estos datos esta mediada por el uso de diversos instrumentos de recolección de datos, de estos destaca el cuestionario, la encuesta, la entrevista y la indexación documental.

Los exploratorios descriptivos (75\%), indican precisiones conceptuales en ciertas variables que están inscritas en el fenómeno de sexting consensuado y no consensuado en poblaciones adolescentes. Sirven para identificar, ponderar y comparar rasgos visibles de conductas en las poblaciones objeto. Finalmente, se presenta un estudio analítico (25\%), cuyo objetico es comparar entre poblaciones de adolescentes jóvenes y adultos emergentes la prevalencia del sexteo consensuado y no consensuado [2].

Tabla II Estudios de acuerdo al alcance de la investigación

\begin{tabular}{lccc} 
Nivel & Frecuencia & \multicolumn{2}{c}{$\%$} \\
\hline Exploratorio descriptivo & & 3 & \multicolumn{2}{c}{75} \\
\hline Analítico & 1 & 25 & \\
\hline Total & 4 & 100
\end{tabular}


Esto indica que se tiende a establecer un marco teórico sustantivo y general. Estos estudios se caracterizan por combinar la perspectiva sincrónica para demostrar y comparar hipótesis de trabajo, junto con una perspectiva diacrónica que indaga mediante el establecimiento de un corte temporal ciertas tendencias investigativas.

\section{Diseño de Investigación}

En cuanto a los diseños de investigación se destacan los de tipo cuasiexperimentales (75\%), cuyo objetivo es el contraste de las hipótesis que guían estos estudios. La principal limitación de estos estudios es la aplicación de estrategias e instrumentos de recolección de datos. La falta de introspección e "intimación" con el sujeto de estudio tiende a ser superada mediante el uso de la técnica de Focus Group" o grupo de control [19]. Debido que se trata de poblaciones de adolescentes juveniles muy heterogéneas que interactúan dentro de la multidimensionalidad de la sexualidad en línea, el grupo de control se acerca a un enfoque medianamente "cualitativo" donde los participantes pueden expresar ideas y opiniones en base a una lista de preguntas.

Esta evidencia nos remite a la consideración expresa que en las investigaciones se utiliza un tipo de muestreo intencional aleatorio como condición para expresar la representatividad de la población objeto de estudio. Aunque la mayoría de los casos revisados declaran limitaciones en la definición de esta población, ven como único recurso disponible el muestreo aleatorio. Ocasionando que los estudios cuasiexperimentales sean estudios con niveles y grado de evidencia moderada $(75 \%)$.

Tabla III Estudios de acuerdo al diseño de investigación

\begin{tabular}{lcc}
\hline Diseño & Frecuencia & \% \\
\hline Cuasi-experimental & 3 & 75 \\
\hline Revisión documental & 1 & 25 \\
\hline Total & $\mathbf{4}$ & $\mathbf{1 0 0 \%}$
\end{tabular}

\section{Relación metodología v/s evidencia}

Revisando la relación metodología vs evidencia, encontramos que las publicaciones examinadas presentan la siguiente descripción: Un porcentaje de $25 \%$ de artículos presenta alta calidad de la evidencia (I.a).- El nivel de evidencia moderada (II.b) agrupa por igual un porcentaje alto de 50\% de los artículos revisados. Un porcentaje de $25 \%$ de artículos presenta nivel de evidencia III. Esta descripción indica que la mayoría de las publicaciones revisadas (75\%) cuentan con interés científico, con niveles de evidencia científica buena y moderada (grados I.a y II.b), procedentes de estudios controlados y cuasiexperimentales. Solo un estudio (25\%) puede ser considerado con un nivel de evidencia III. El 100\% de los artículos revisados coincide con nivel A y nivel B, con niveles de evidencia buena y moderada (A y B). En otras palabras, la norma en este tipo de investigaciones es de tipo empírico inductiva. 
Tabla IV Resumen de nivel de evidencia y grado de recomendación

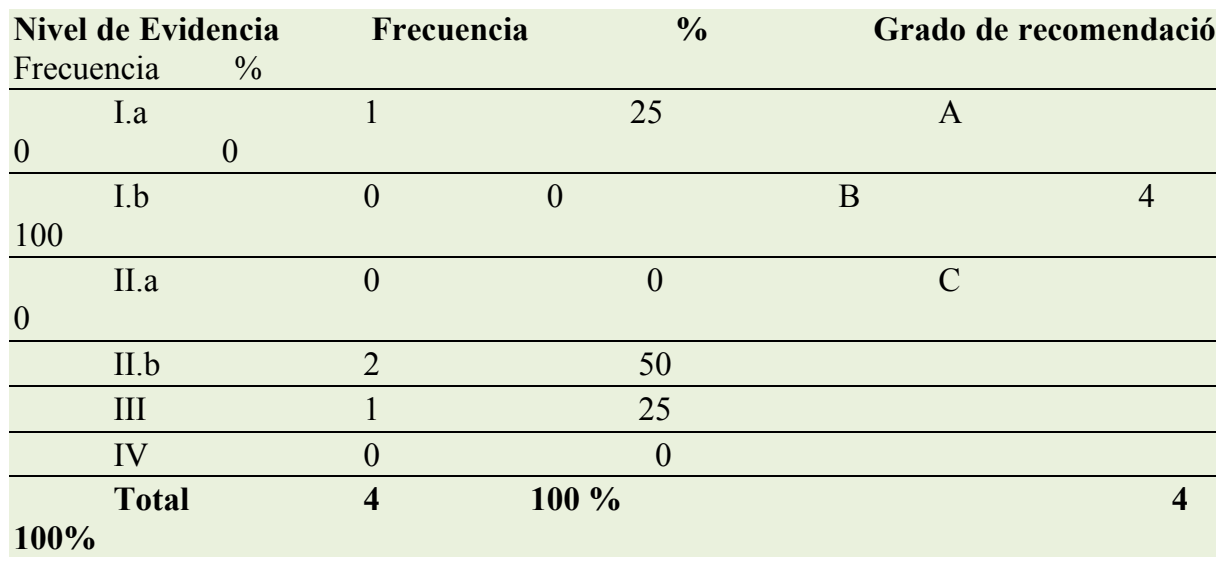

\section{ANÁLISIS DE TENDENCIAS TEÓRICAS}

En la categoría Sexualidad Virtual Normativa y Dual, existe una investigación cuyo interés es caracterizar la sexualidad adolescente (temprana y tardía) y la práctica del sexting, tanto consensuado como no consensuado. [2]

En este sentido se destaca que el comportamiento de sextear sería probablemente inofensivo cuando se consiente mutuamente, pero puede ser problemático (y potencialmente ilegal) si los sextos se envían sin consentimiento. [2, p.13]. Lo anterior se puede conectar a que la sexualidad adolescente temprana y tardía, expresada en la práctica del sexting, comporta esta misma dualidad intrínseca: es decir cuando se procede sin consentimiento y cuando se procede con el mismo. De aquí su carácter normativo.

En un caso supone que puede acarrear riesgos del tipo social y mental, y en el otro este mismo riesgo se presenta minimizado absolutamente. Esto se expresa en la afirmación de Mori et. al [2]cuando indica que se ha cuestionado la estigmatización del sexting ya que este puede ser una expresión moderna normativa de la sexualidad, más que un comportamiento claramente desviado.

Esta noción implica necesariamente considerar y relevar para investigaciones futuras los factores de contexto adicionales que permitirían conocer las condiciones en las que comportamiento se da [7]. Además, en ese mismo sentido, corresponde incluir esta consideración en todos aquellos modelos socioeducativos que pueden servir de orientación en el campo de la sexualidad adolescente.

En la categoría Integración en la Sexualidad Adolescente de los Impactos Positivos del Sexting Consensuado se incluyen las investigaciones que obtuvieron un moderado nivel de evidencia y cuyo interés es demostrar que la formación de ". . . nuevas prácticas afectivas virtuales requiere redefinir el paradigma tradicional de la educación sexual, integrando la privacidad en la era digital, la toma de decisiones libre, consciente y crítica, y el establecimiento de relaciones consensuales como aspectos clave en todos los programas.[14,p. 9]

De igual modo, Fix et.al [19] también enfatiza sobre "...la necesidad de integrar los comportamientos de sexteo de los adolescentes en los planes de estudio integrales de educación sexual y de desarrollar contenido educativo sobre los comportamientos de sexteo de los adolescentes para adolescentes y cuidadores en formatos de folletos en línea e imprimibles." [19, p. 1]

En relación a la categoría "Sexting consensuado y reciproco relacionado con la satisfación sexual, afectividad y aceptación" encontramos una publicación [11] que si bien menciona las consecuencias y los aspectos negativos y positivos de la práctica del sexting, introduce una perspectiva relativista al considerar que "... en términos de implicaciones prácticas, estos hallazgos sugieren que se necesita más discusión y educación con respecto a 
la definición de coerción sexual y la negociación del consentimiento dentro de las experiencias sexuales en línea, ya que es un proceso complicado." [11, p. 2]

Esta perspectiva relativista asume que las poblaciones objeto de estudio no son homogéneas. De modo que la heterogeneidad obliga particularizar los enfoques en el diseño de las investigaciones. Prueba de ello es el hecho de que de acuerdo a Fix et.al y Cornelius, et. al $[11,19]$ se encontró que existe una dualidad en la que se informa que las motivaciones, expectativas y resultados inmediatos del sexteo son en su mayoría positivos, pero los correlatos de relación suelen ser negativos

\section{DISCUSIÓN Y CONCLUSIONES}

Esta revisión recogió las publicaciones desde el año 2019 al 2020 que se ajustan a los criterios de inclusión (4 artículos), para describir qué indica la literatura en relación a sexting consensuado en el marco de la cibersexualidad adolescente. Se considera que esta investigación, debido a lo actualizado de la literatura que revisa y a la escasez de investigaciones que existen del tema especifico, podría ser de interés para los/as investigadores/as y aportará a la generación de un conocimiento más profundo de la temática del sexting.

En general, y de acuerdo con los resultados de los artículos revisados, los estudios empíricos son los más frecuentes dentro de la totalidad de las investigaciones. Los documentales están representados en una menor proporción en comparación a los primeros. Todos los estudios alcanzaron nivel de evidencia moderada y resultaron ser del tipo exploratorio descriptivo y descriptivo analítico. En ningún caso hubo del tipo explicativo. Los diseños de estudios fueron de tipo documental y cuasi-experimentales. Con un nivel de evidencia I.a, II.b.y III.

Los/as investigadores/as priorizaron la encuesta, la entrevista, el cuestionario y el análisis de observación como instrumentos de recolección de datos frente la revisión teórica.- Sólo se identificaron tres tendencias investigativas.

Se detectó un alto grado de coherencia entre los métodos y las teorías desarrolladas.

El análisis teórico y metodológico realizado en estos estudios y según las tendencias obtenidas, presenta tres categorías en las cuales se agrupan los diferentes artículos. La primera tiene que ver con Sexualidad virtual normativa y dual. [2].La segunda hace referencia a la integración en la sexualidad adolescente de los impactos positivos del sexting consensuado. [14 y 19]. Y la tercera, indica el sexting consensuado y reciproco relacionado con la satisfación sexual, afectividad y aceptación. [ 11]

Agrupando estas tres categorías podemos discriminar dos supuestos de orden sustantivos (teórico y metodológico) que delimitan el significado de la práctica del sexteo consensuado: Uno que demuestra que el carácter de la cibersexualidad adolescente no constituye un modelo de interpretación único; por el contrario, su comprensión dependería del examen de los componentes contextuales de las poblaciones objeto. Otro supuesto es que toda nueva interpretación o perspectiva debe ser incorporada en el marco de la prevención y la educación.

La primera categoría nos demuestra que la práctica del sexting no sólo forma parte de la nueva sexualidad adolescente, sino que también es un hecho concreto normalizado dentro de esa misma sexualidad. La segunda categoría muestra que la responsabilidad de diseñar, conducir e impartir una política de prevención y educación holística o global en torno a la sexualidad adolescente fundamentalmente recae en la instrucción formal, enfocada en la importancia del respeto a la privacidad, el consentimiento y la promoción de la ética sexual tal como lo indica Dobson citador en 0jeda [20].

Y en la tercera categoría hay evidencia de una perspectiva de tipo interdisciplinaria que rescata un enfoque relativista y particularista de la sexualidad adolescente.

Como colofón de este análisis es importante destacar que más allá de las derivaciones de orden metodológico y teórico que estas categorías denotan, hay que señalar que existe un alto nivel de impacto en los contenidos éticos, legales y sociales asociados al sexting en adolescentes. Normalizar la práctica del sexting; aceptar su dualidad; Io negativo y lo positivo [21,] incorporar este hecho en los planes de prevención y educación [22] y aceptar que lo universal no es la regla sino que todo es dependiente de los contextos socioculturales e históricos, representa introducir una crítica a lo socialmente aceptado. 


\section{REVISIÓN SISTEMÁTICA ACERCA DE LAS TENDENCIAS INVESTIGATIVAS DEL SEXTING CONSENSUADO EN EL MARCO DE LA CIBERSEXUALIDAD ADOLESCENTE}

Ante este cuadro lo mas idóneo es tener una base conceptual de cómo proceder ante la nueva sexualidad en línea; fortalecer la intimidad de las personas a través de la generación de información científica y objetiva [23], no retraer la actuación de una sexualidad libre bajo estereotipos de género y de prohibición moral, ya que la cibersexualidad está irrumpiendo como un aspecto definitorio de la sexualidad adolescente.

Las nuevas formas de socialización y comunicación y la comprensión de esta "extimidad" [24] nos hace preguntarnos acerca del significado que la intimidad tiene para los jóvenes y considerar la pertinencia de abandonar el presupuesto y lugar teórico de referencia de todo lo que tradicionalmente se considera parte del reino de la privacidad y estar abiertos a que las respuestas traigan nuevas definiciones sobre lo íntimo, lo privado y lo público. La experiencia que presentan los y las jóvenes se caracterizan por una resignificación de este concepto en el mundo virtual, lo que no necesariamente se expresa en otros contextos.

Si bien existe un cierto consenso en que el sexteo conlleva situaciones de riesgo, [7] para los adolescentes, la inconsciencia de riesgo potencial de las redes sociales para la práctica del sexting se puede deber a que nacieron con ellas y se imitan a través de éstas" [26] este fenomeno se considera normalizado por ellos y es una forma propia que este rango etario tiene de entender y experimentar las relaciones de pareja y la sexualidad.

\section{REFERENCIAS BIBLIOGRÁFICAS}

Wolak J, Finkelhor D. Sexting: A Typology. Crimes Against Children Research Center [Internet]. 2011 [citado 01 septiembre 2020] Sp];(. ):4-6. Disponible en: http://www.unh.edu/ccrc/pdf/CV231_Sexting\%20Typology\%20Bulletin

Mori C, Cooke J, Temple J, Ly A, Lu Y, Anderson N et al. The Prevalence of Sexting Behaviors Among Emerging Adults: A Meta-Analysis. Archives of Sexual Behavior. 2020; 49 (4):1103-1119.

Krieger M. Unpacking "Sexting": A Systematic Review of Nonconsensual Sexting in Legal, Educational, and Psychological Literatures. Trauma, Violence, \& Abuse. 2016;18 (5):593-601.

3. Van Ouytsel J, Lu Y, Ponnet K, Walrave M, Temple J. Longitudinal associations between sexting, cyberbullying, and bullying among adolescents: Cross-lagged panel analysis. Journal of Adolescence. 2019;73:36-41.

Maas MK, Bray BC, Noll JG. Online Sexual Experiences Predict Subsequent Sexual Health and Victimization Outcomes Among Female Adolescents: A Latent Class Analysis. J Youth Adolesc. 2019 May; 48(5):837-849. doi: 10.1007/s10964-019-00995-34.

Sanhueza, C. Revisión sistemática acerca de las tendencias investigativas sobre sextig adolescente en la última década. Conf. Cephalal. et Neurol. 2020 dic; 30 № 3: 139-147. https://mattiolihealth.com/wpcontent/uploads/2020/12/cover-and-index-Conf-Cephalal-et-Neurol-3-20.pdf

Scheechler, C. Aspectos fenomenológicos y políticos-criminales del sexting. Aproximación a su tratamiento a la luz del Código Penal chileno. Política Criminal. 2019 jul; 14 (27): 376-418. https://dx.doi.org/10.4067/S0718-33992019000100376

Pai M, McCulloch M, Gorman J, Pai N, Enanoria W, Kennedy G, Tharyan P, Colford J Jr. Systematic reviews and meta-analyses: an illustrated, step-by-step guide. Natl Med J India. 2004 Mar-Apr; 17(2):86-95. PMID: 15141602

Alonso C, Romero E. Conducta de sexting en adolescentes: predictores de personalidad y consecuencias psicosociales en un año de seguimiento. Anales de Psicología. 2019;35(2):214-224.

González R, Veray A, Adorno R, et al. La práctica del sexting y la satisfacción en la relación de pareja: un estudio exploratorio. Rev Elec Psic Izt. 2018; 21(3):886-903.

Cornelius T, Bell K, Kistler T, Drouin M. Consensual Sexting among College Students: The Interplay of Coercion and Intimate Partner Aggression in Perceived Consequences of Sexting. International Journal of Environmental Research and Public Health. 2020; 17(19):7141.

8. Del Prete A, Redon Pantoja S. Las redes sociales virtuales: Espacios de socialización y definición de identidad. Psicoperspectivas Individuo y Sociedad. 2020; 19(1). 
Giraldo León C. Cibercuerpos: Ios jóvenes y la sexualidad en la posmodernidad. Actualidades Investigativas en Educación. 1970; 13 (1).

Soriano-Ayala E, C. Cala V, Dalouh R. Adolescent Profiles According to Their Beliefs and Affinity to Sexting. A Cluster Study. International Journal of Environmental Research and Public Health. 2020; 17(3):1087.

Valido A, Espelage D, Hong J, Rivas-Koehl M, Robinson L. Social-Ecological Examination of Non-Consensual Sexting Perpetration among U.S. Adolescents. International Journal of Environmental Research and Public Health. 2020; 17(24):9477.

Villada J, Chaves L, Jaramillo C. Revisión sistemática sobre habilidades de pensamiento en el aula. Estudios pedagógicos [Internet]. 2016 [citado 28 septiembre 2020]; vol.42 (2): 365-377. doi.10.4067/S071807052016000200021

Cooper H. Research synthesis and meta-analysis: a step- by-step approach. 4th ed. California: Kinight, 2007.

Moraga J, Cartes R. Pautas de Chequeo, parte II: Quorom y Prisma. Rev Chil Cir [Internet]. 2015 Jun [citado 18 septiempbre de 2020]; 67 (3): 325-330. Disponible en: doi.org/10.4067/S0718-40262015000300015.

Fix R, Assini-Meytin L, Harris A, Letourneau E. Caregivers' Perceptions and Responses to a New Norm: The Missing Link in Addressing Adolescent Sexting Behaviors in the U.S. Arch Sex Behav. 2021 Feb; 50(2):575588. doi: 10.1007/s10508-020-01704-z. Epub 2020 Apr 26. PMID: 32337659.

Ojeda M, del-Rey R, Walrave M, Vandebosch H. Sexting in adolescents: Prevalence and behaviours. Comunicar. 2020; 28 (64):9-19. 19. https://doi.org/10.3916/C64-2020-01

Englander E. What Do We Know About Sexting, and When Did We Know It?. Journal of Adolescent Health. 2019; 65(5):577-578. Englander, E. (2019). What do we know about sexting, and when did we know it? Journal of Adolescent Health, 65(5),

577-578. https://doi.org/10.1016/j.jadohealth.2019.08.004

McEachern, A, McEachern- Ciattoni, R, Martin, F. Sexting: New challenges for schools and professional school counselors. Journal of School Counseling 10(20), 1-28.

Montero A. Educación sexual: un pilar fundamental en la sexualidad de la adolescencia. Rev. méd. Chile [Internet]. 2011 Oct [citado 2021 Mayo 20]; 139(10): 1249-1252. Disponible en: http://www.scielo.cl/scielo.php?script=sci_arttext\&pid=S0034

Sibilia P. La intimidad como espectáculo. Comunicación y Medios. 2012; 0(22). (2008).

Fajardo M, Gordillo M, Regalado, A. Sexting: Nuevos Usos De La Tecnología Y La Sexualidad En Adolescentes. International Journal of Developmental and Educational Psychology [Internet]. 2013;1 (1):521-533. Recuperado de: https://www.redalyc.org/articulo.0a?id=349852058045

Arias M, Buendía Eisman L, Fernández F. Grooming, Ciberbullying y Sexting en estudiantes en Chile según sexo y tipo de administración escolar. Revista chilena de pediatría. 2018;(ahead):0-0. 
